\title{
System design of terabit-per-second capacity low earth orbit optical feeder links
}

Paul Shubert, Jim McNally

Paul Shubert, Jim McNally, "System design of terabit-per-second capacity low earth orbit optical feeder links," Proc. SPIE 11852, International Conference on Space Optics - ICSO 2020, 1185216 (11 June 2021); doi:

$10.1117 / 12.2599228$

SPIE Event: International Conference on Space Optics - ICSO 2021, 2021, Online Only 


\section{International Conference on Space Optics-ICSO 2020}

Virtual Conference

30 March-2 April 2021

Edited by Bruno Cugny, Zoran Sodnik, and Nikos Karafolas
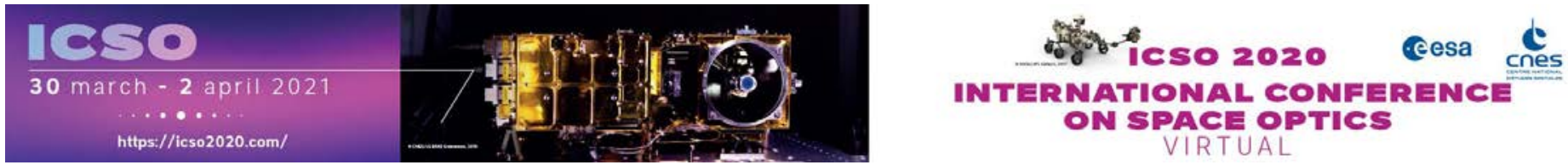

\section{System design of terabit-per-second capacity low earth orbit optical feeder links}

\section{Cesa isoporecestings denes}




\title{
System design of Terabit-per-second capacity low earth orbit optical feeder links \\ Paul Shubert, Jim McNally \\ Applied Technology Associates, 1300 Britt St SE, Albuquerque, NM USA 87123-3353 \\ ${ }^{a}$ StratTHNK Associates, LLC
}

\begin{abstract}
Future space-borne synthetic aperture radar, hyper-spectral imaging, and high spatial and temporal resolution imaging will place increasing demands on space network data throughput. It is expected that space data relay systems will require capacities of multiple Terabits per second (Tbps). Free-space optical communication is likely the only technology capable of supporting these data demands. A previous study ${ }^{1}$ has shown that implementing these high-capacity optical feeder links through a traditional geostationary constellation will pose significant development challenges. Meshed satellite constellations in low earth orbit (LEO), operating at significantly shorter ranges, were shown to have the potential to support Tbps feeder links using the technical capabilities of current free-space optical communication systems. Meshed LEO constellations, however, provide unique challenges, including complex constellation maintenance, dynamic meshing and data routing, and short contact periods with ground stations.

We review the design requirements for high capacity LEO relay optical terminals and satellites. The global coverage and ground station downlink opportunities of constellation options are evaluated to develop constellation designs utilizing the minimum number of satellites while providing required feeder link performance. The constellations are designed to provide mesh links with Tbps feeder link capabilities using optical terminals comprised of components within current state-of-the-art and with demonstrated capabilities. These meshed feeder link constellations were then modelled to demonstrate the ability of a LEO constellation to gather Tbps of data over a global network, relay this information around the constellation, and downlink this data to a limited number of ground stations.
\end{abstract}

Keywords: free-space optical communication, data relay network, LEO constellations

\section{INTRODUCTION}

Free-space optical communication systems have held the promise of higher data rates, lower size, weight, and power ( $\mathrm{SWaP}$ ), and lower cost per bit when compared to radio frequency (RF) communications systems. In practice, however, in response to market and competitive pressure, the state-of-the-art for RF communication systems has continued to advance, with satellite throughput capacities in the Ka-band approaching 500 gigabits per second (Gbps) ${ }^{2,3}$. To be competitive, optical communication systems will need to advance their performance expectations to meet or exceed the capabilities available at RF.

Advances in space sensing capabilities, including advanced synthetic aperture radars, hyper-spectral imaging, and highspatial and temporal resolution imaging, will require space relay networks with ever-increasing data capacity. These systems will push capacity requirements beyond the capabilities of even advanced RF systems. Optical communications, with theoretical capacities of multiple terabits per second (Tbps), will be required to support these applications.

Commercial development has pushed the bandwidth capacity of terrestrial fiber systems to theoretical limits. Coherent transceiver systems with 100 to $200 \mathrm{Gbps}_{\text {capacities }}{ }^{4}$ operating with dense wavelength division multiplexing (DWDM) can fully utilize the multi-Tbps bandwidth of C-band erbium doped fiber amplifiers (EDFA).

A space relay system is expected to receive data from numerous sources, and then aggregate and transmit this data around a global relay constellation, downlinking the data to users through a system of ground terminals. Several options exist for the relay constellation, including a traditional network of linked geostationary (GEO) satellites, and a mesh network of satellites in low-earth orbit (LEO). Our previous study ${ }^{1}$ shows that implementing these high-capacity optical feeder links through a traditional geostationary constellation will pose significant development challenges. Meshed satellite constellations in low earth orbit (LEO), operating at significantly shorter ranges, were shown to have the potential to support Tbps feeder links using the technical capabilities of current free-space optical communication systems. 


\section{LEO CONSTELLATION DESIGN}

Table 1 summarizes the system designs for high-capacity ( $>1 \mathrm{Tbps})$ optical relay networks. LEO networks operate at lower altitudes and shorter ranges, with many more satellites required to provide global coverage. This mesh of satellites then reduces the capacity requirements for individual inter-satellite links. The net result is that the performance requirements for a LEO-based optical terminal are significantly relaxed compared to the stringent requirements imposed by the higher capacity and longer operating range of a GEO relay network.

Table 1. Notional high-capacity optical network LEO and GEO terminal designs.

\begin{tabular}{|l|r|r|}
\hline \multicolumn{1}{|c|}{ Parameter } & \multicolumn{1}{c|}{ LEO } & \multicolumn{1}{c|}{ GEO } \\
\hline Aperture & $15 \mathrm{~cm}$ & $30 \mathrm{~cm}$ \\
\hline Tx Power & $10 \mathrm{~W}$ & $100 \mathrm{~W}$ \\
\hline Constellation Size & $50-200$ & 8 \\
\hline Ground Terminal Diameter & $40 \mathrm{~cm}$ & $80 \mathrm{~cm}$ \\
\hline Cross-Link Capacity & $500 \mathrm{Gbps}$ & $1 \mathrm{Tbps}$ \\
\hline Space-Ground Capacity & $2 \mathrm{Tbps}$ & $4 \mathrm{Tbps}$ \\
\hline
\end{tabular}

Examining these designs in detail reveals that LEO terminal designs are well within current capabilities. Space optical terminals with 10 to $15 \mathrm{~cm}$ apertures, and their corresponding tracking and stabilization systems, have been demonstrated ${ }^{5}$, and similar systems with data rates in excess of $1 \mathrm{Gbps}$ are currently in development, with space demonstrations planned in the near future ${ }^{6,7}$. Similarly, the $10 \mathrm{~W}$ transmit laser power requirement for the LEO terminal system is currently available in commercial versions ${ }^{8}$. Higher power amplifiers also begin to suffer from significant crosstalk and parasitic losses from four-wave mixing between DWDM channels ${ }^{9}$. For these reasons, high-capacity LEO relay systems have significantly less developmental risk compared with their GEO counterparts.

Providing a high-capacity LEO data relay system with global coverage requires a mesh of optical links between the satellites in the constellation. Generally, these constellations consist of satellites evenly distributed in a number of orbital planes at high inclinations, as shown in Figure 1. Satellites support optical links to the satellites in-plane leading and trailing each satellite, and across planes to satellites nominally "in-phase" in the current orbit to create a stable mesh network. The pointing requirements for this mesh geometry have been analyzed by Miller ${ }^{10}$.

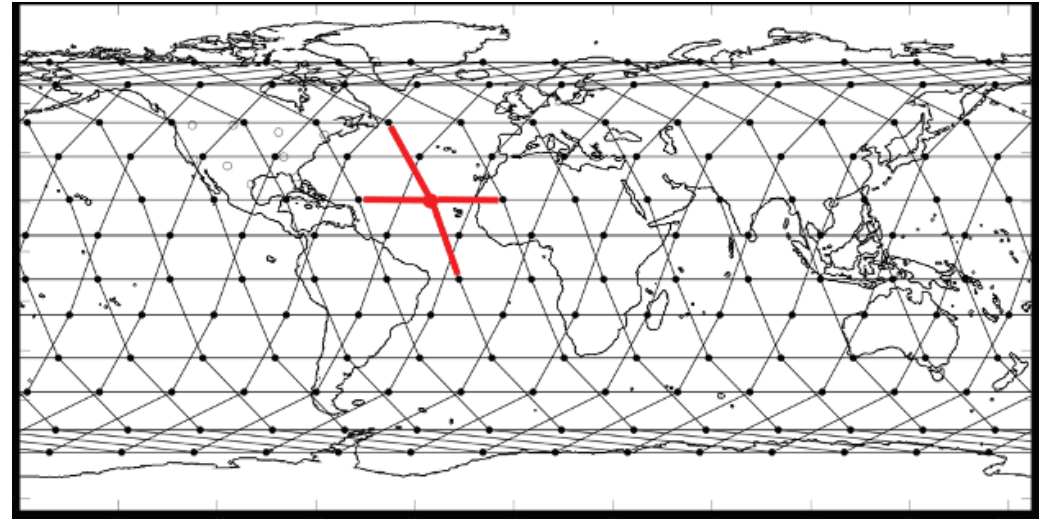

Figure 1. Relay constellation consists of satellites spaced in high inclination orbits with in-plane and cross-plane links between satellites.

In addition to relaying data around the constellation, the network is expected to downlink this data to ground stations. The LEO constellation poses significant challenges in this area, as ground station visibility from any one satellite is very limited. The siting requirements for the ground stations also provide conflicting requirements. Widely dispersed ground stations provide diversity for weather conditions, but it may be desirable to limit ground system locations to a specific geographic area, such as the continental United States (CONUS) as shown in Figure 2, or the European Union (EU). The baseline terminal design also only supports 2 Tbps of downlink capacity through its four $500 \mathrm{Gbps}$ crosslinks, so more satellites in view will be required to support networks with higher data capacity requirements. 


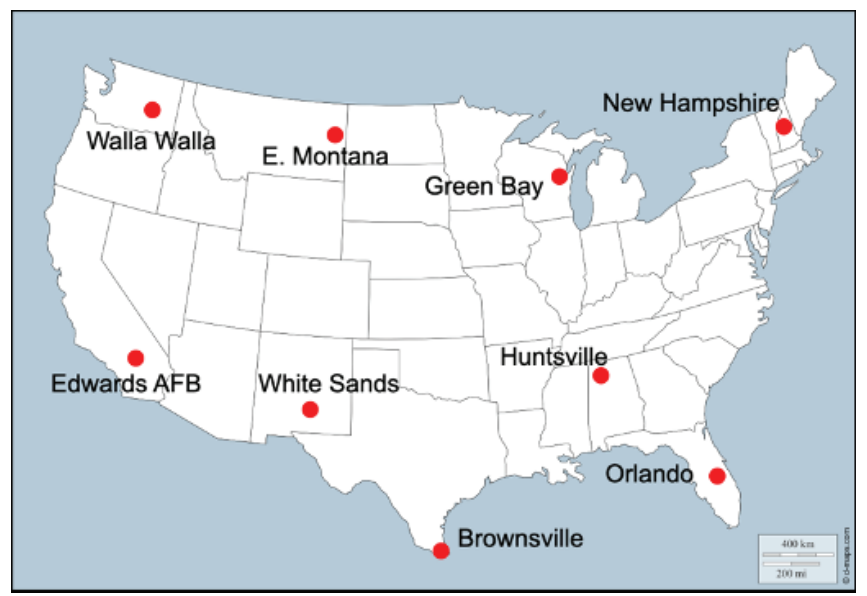

Figure 2. Representative ground stations limiting downlink opportunities to the continental United States (CONUS).

Ground terminal and satellite visibility depends on a number of factors, including the specific constellation geometry, number of planes, number of satellites, orbital inclination, etc., and ground station latitude. A simulation was developed to analyze the downlink opportunities available for a number of ground stations as a function of various constellation geometries. The effects of latitude and geographic constraints was modeled by choosing a number of sites within the continental United States, as shown in Figure 2. These sites were simply chosen to maximize geographic diversity within the siting constraints.

A number of different constellation configurations were then modeled to determine the minimum number of ground stations visible, and the minimum number of satellites capable of establishing downlinks for all satellite and earth rotation conditions. The results of this simulation are shown in Table 2. While it is possible to provide global mesh coverage with as little as 60 satellites, 6 planes with 10 satellites each, the simulation shows that these minimum configurations can be limited to visibility from 2 or 3 ground stations out of the 9 total, and of course these particular ground stations may obscured due to weather. Larger constellations increase the availability of ground stations, resulting in greater site diversity and increased probability that ground stations are available to downlink data from at least two satellites.

The Table also illustrates that smaller constellations may have 3 or fewer satellites capable of downlinking data. Again, if the ground stations visible to these satellites are obscured by clouds, then the constellation would be limited to less than 2 Tbps of data throughput.

With the baseline optical terminal design, link capacity, downlink geometry constraints, and data throughput requirements, constellation geometries of about 200 satellites would be required to reliably support the overall system requirements for relay networks requiring greater than 2 Tbps of data throughput.

Table 2. Minimum number of CONUS ground stations visible and minimum number of satellites for various constellation geometries.

\begin{tabular}{|c|c|c|c|c|c|c|c|}
\hline \multirow{2}{*}{$\begin{array}{c}\text { Sats } \\
\text { per } \\
\text { Plane }\end{array}$} & \multicolumn{7}{|c|}{ Number of Planes } \\
\cline { 2 - 8 } & $\mathbf{6}$ & $\mathbf{8}$ & $\mathbf{1 0}$ & $\mathbf{1 2}$ & $\mathbf{1 4}$ & $\mathbf{1 6}$ & $\mathbf{1 8}$ \\
\hline $\mathbf{1 0}$ & $2 / 1$ & $2 / 2$ & $4 / 3$ & $5 / 4$ & $5 / 4$ & $5 / 5$ & $5 / 6$ \\
\hline $\mathbf{1 2}$ & $3 / 2$ & $3 / 2$ & $5 / 4$ & $5 / 4$ & $6 / 5$ & $7 / 6$ & $7 / 7$ \\
\hline $\mathbf{1 4}$ & $3 / 2$ & $4 / 2$ & $5 / 4$ & $6 / 4$ & $6 / 5$ & $7 / 6$ & $7 / 7$ \\
\hline $\mathbf{1 6}$ & $5 / 2$ & $5 / 3$ & $7 / 6$ & $7 / 6$ & $8 / 8$ & $9 / 9$ & $9 / 10$ \\
\hline $\mathbf{1 8}$ & $5 / 3$ & $5 / 4$ & $7 / 6$ & $7 / 7$ & $8 / 9$ & $9 / 10$ & $9 / 11$ \\
\hline $\mathbf{2 0}$ & $6 / 3$ & $6 / 4$ & $8 / 7$ & $8 / 9$ & $9 / 10$ & $9 / 11$ & $9 / 14$ \\
\hline
\end{tabular}




\section{MESH SIMULATION}

A meshed LEO satellite constellation appears to have enough throughput capacity to collect data over the globe, relay the data around the constellation, and downlink the data to ground stations. Practical implementation of this network, however, will have to accommodate the fact that data is not collected uniformly over the mesh, that the mesh density over the earth in not uniform, and that the collected data must be concentrated in the mesh for download to a geographically limited area. Figure 3 illustrates the differences in satellite density which occurs in a global mesh of LEO satellites in high inclination orbits, which results in higher satellite density over the sparsely populated polar regions.

A simulation of the LEO relay network was implemented to evaluate whether a meshed LEO constellation would be capable of gathering the required amount of data distributed non-uniformly over the earth and relaying it to ground stations. The simulation assumed a distribution of data based on population density and modeled the limited link capacity between satellites, as well as the overall orbital dynamics of the constellation.

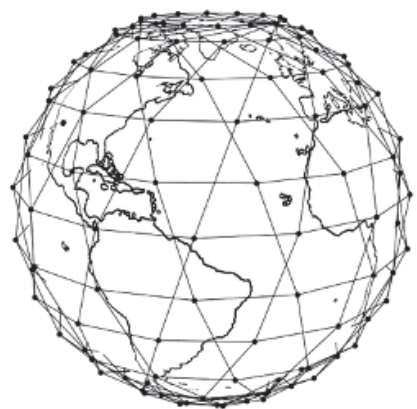

Figure 3. LEO mesh constellation results in higher concentration of satellites in polar regions.

\subsection{Data Loading}

A model of data loading over the earth was developed to examine the ability of the LEO mesh to adapt to relaying nonuniform amounts of data around the constellation. The model assumes that the amount of data generated by any specific area on the earth is roughly proportional to population density, although it was assumed that all areas would generate some amount of data, climatological data, for example. Initial input to the data model consisted of a map of population density, shown in Figure $4^{11}$. This data was used to assign areas on the earth one of five data load categories: ocean, sparsely populated land, and three categories of populated areas. The density of data produced by each category was then set to be roughly proportional to the population density, and to result in a total data load of $4 \mathrm{Tbps}$ across the earth. This data density is shown in Table 3 .

The data from each area was aggregated into a 36 x 72 element grid, shown in Figure 5, to simplify modelling. Mesh data loading was then modelled by taking the total data in each grid point and transferring it to the nearest satellite.

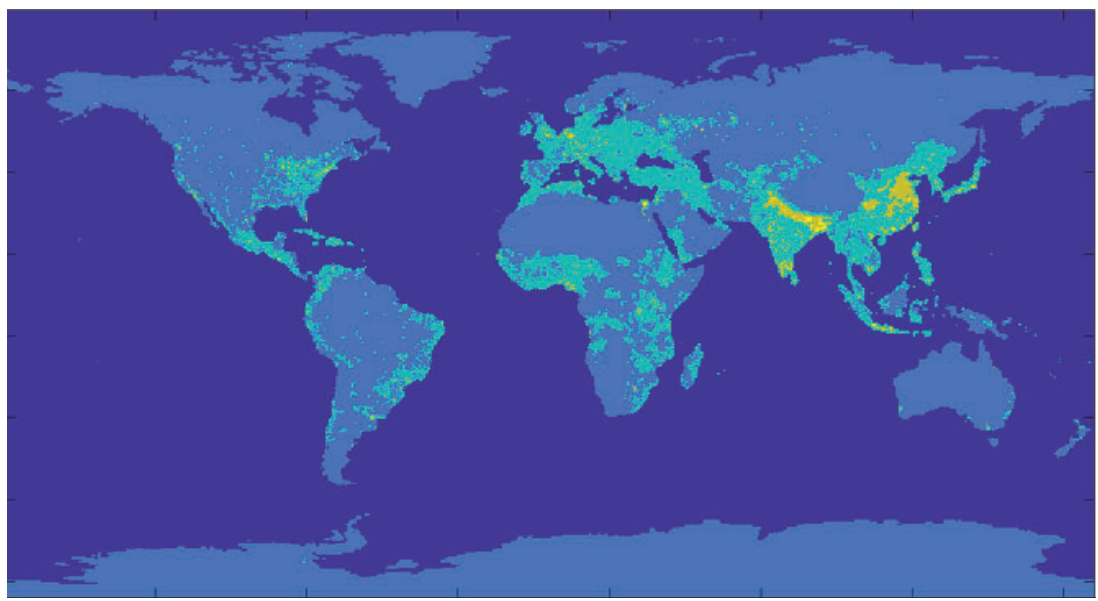

Figure 4. Population density (NASA 2000) 
Table 3. Data generated from each surface zone resulting in 4 Tbps total data load.

\begin{tabular}{|l|c|c|}
\hline \multirow{2}{*}{ Surface Composition } & \multicolumn{2}{|c|}{ Data Generated } \\
\cline { 2 - 3 } & $\mathbf{k b i t s} / \mathbf{s} / \mathbf{k m}^{\mathbf{2}}$ & $\mathbf{~}^{\mathbf{2}} / \mathbf{b i t} / \mathbf{s}$ \\
\hline Ocean & 1.8 & 555 \\
\hline Sparsely Populated & 17.5 & 57 \\
\hline Populated & 35.0 & 28 \\
\hline Densely Populated & 52.6 & 19 \\
\hline Extreme Density & 70.0 & 14 \\
\hline
\end{tabular}

\subsection{Meshing Algorithm}

Once the total data collected by each satellite was determined, the simulation transferred this data around the constellation to a satellite within the view of a ground station. The amount of data transferred on each inter-satellite link is limited to the $500 \mathrm{Gbps}$ of the design cross-link capability, and the maximum amount of data which can be downloaded to the ground station from a single satellite is limited to 2 Tbps. To relay and downlink 4 Tbps of data requires an adaptive meshing algorithm to route data around congested links and to links which have available capacity.

The meshing algorithm implemented a "drain" model. A data chain was initiated from each satellite in view of a ground station, which had a total capacity of $2 \mathrm{Tbps}$, divided into four $500 \mathrm{Gbps}$ inter-satellite links. The data load of each satellite connected by a direct link to the downlink satellite was then subtracted from the link chain capacity, and the process repeated for each direct link of this second satellite.

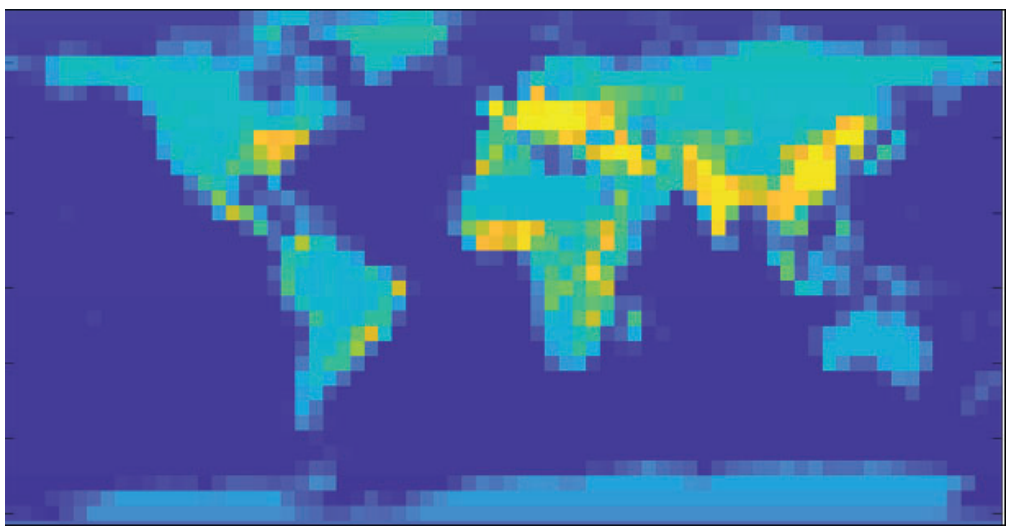

Figure 5. Model data density.

This process then continued through the satellite constellation, with each satellite, in turn, sending its data load down the chain, if sufficient capacity was available. Because each satellite could be reached by multiple chains, this resulted in a satellite's data load sent along the nominally shortest path with available capacity.

This first-order data drain algorithm does not ensure that all of the data in the constellation would find a path, as occasionally the direct chain path would fill to its $500 \mathrm{Gbps}$ limit. After the constellation was meshed by the first drain algorithm, any satellites with a remaining data load were identified, and the nearest satellite with data capacity was located. The remaining data load was then relayed across chains to arrive at a satellite with remaining capacity. This fairly simple set of algorithms was shown to be able to successfully relay the 4 Tbps of data around the constellation.

\subsection{Simulation Results}

The simulation was performed for various constellation geometries. While the analysis was not exhaustive, the simulation confirmed that constellation geometries which ensured that sufficient downlink capacity existed could be meshed to relay the non-uniform simulated data load. 


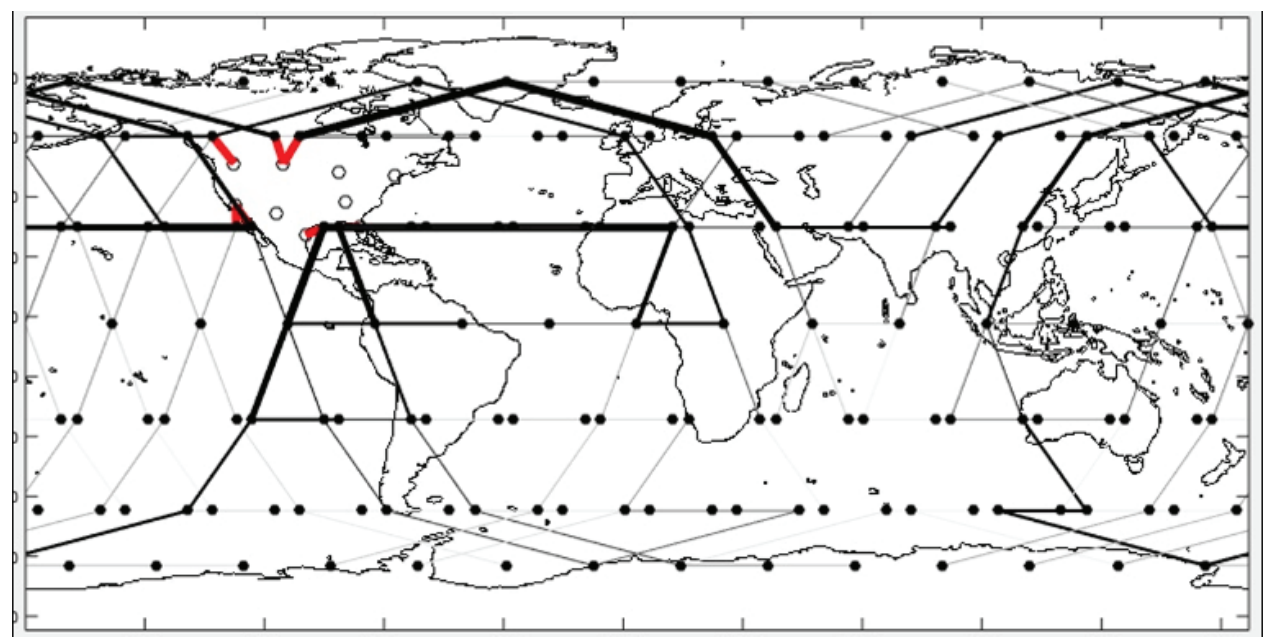

Figure 6. Crosslink loading in a 4 Tbps LEO data relay mesh.

An example data loading result is shown in Figure 6. The figure shows that meshing algorithm is capable of routing the 4 Tbps of collected data over the 500 Gbps crosslinks of the LEO network, with the bulk of collected data efficiently around nominally great-circle paths, with most data transferred over either polar paths or direct paths to CONUS over the Atlantic and Pacific oceans.

\section{CONCLUSIONS}

Meshed satellite constellations in LEO, operating at significantly shorter ranges than their GEO counterparts, are shown to have the potential to support Tbps feeder links using the technical capabilities of current free-space optical communication systems. These systems require relatively complex data routing compared with GEO systems, but this simulation has demonstrated LEO relay networks are capable of efficiently collecting unevenly distributed data and relaying this to geographically constrained ground stations.

The relatively large numbers of LEO satellites required will impact the overall cost of the system. Ignoring the significant costs required to develop high-capacity GEO optical terminal systems, the costs of establishing LEO- and GEO-based optical relay networks may be estimated from scaling relative terminal size and complexity and launch costs. An estimate of these relative costs is shown in Table 4.

Because of their smaller aperture size and lower complexity, the cost and weight of a LEO terminal spacecraft is expected to be significantly less than its GEO counterpart. The relative cost to orbit per kilogram is also significantly less to LEO. However, the large number of spacecraft required by the LEO constellation can easily offset these savings. As shown in the table, relative costs between LEO and GEO relay constellations are sensitive to the number of spacecraft required.

Constellation size may be minimized if site availability can be increased. This can be accomplished by using airborne "ground" terminals, such as high-altitude drones or balloons, that can operate above cloud cover. LEO-based systems facilitate this option by requiring smaller ground terminal apertures, on the order of $20 \mathrm{~cm}$ or less. Site availability can also be increased through global site diversity, if geographic constraints on ground terminal location can be lifted.

Table 4. Estimated costs of LEO vs. GEO relay constellations

\begin{tabular}{|l|c|c|c|}
\hline \multicolumn{1}{|c|}{ Parameter } & \multicolumn{2}{c|}{ LEO } & GEO \\
\hline Relative Terminal Cost & \multicolumn{2}{c|}{1} & 5 \\
\hline Relative Cost to Orbit per kg & \multicolumn{2}{|c|}{1} & 6 \\
\hline Terminal Mass & \multicolumn{2}{|c|}{$300 \mathrm{~kg}$} & $500 \mathrm{~kg}$ \\
\hline Relative Launch Cost & \multicolumn{2}{|c|}{1} & 10 \\
\hline Number of Satellites & 50 & 200 & 8 \\
\hline Total Terminal Costs & 1.25 & 5 & 1 \\
\hline Total Launch Costs & 0.6 & 2.5 & 1 \\
\hline
\end{tabular}


The constellation cost can also be reduced by reducing the relative cost of the LEO optical terminals. Both the smaller aperture and reduced data throughput of the LEO terminals aids this, but the larger constellation size and corresponding larger number of terminals required may also allow mass production methods to be brought into play to reduce terminal cost.

Operation in the LEO environment is also less stressing, with significantly reduced radiation requirements compared with GEO, and the opportunity for replenishment is greater, which allows greater flexibility in the qualification of standard telecom components for use in the free-space communication terminals.

Meshed LEO relay constellations may therefore be an alternative to GEO networks for global data routing. The shorter ranges required for LEO constellation meshing allow multiple Tbps systems to be deployed in the near term using existing free-space optical communication technologies. LEO networks offer lower costs to orbit, a less stressing radiation environment, and more frequent replenishment, allowing the network to more easily adapt to technology advancements.

\section{REFERENCES}

[1] Shubert, P., Guthrie, R., Garnham, J., McNally, J., "System Design of a High Capacity Optical Relay Network," IEEE ICSOS, Portland, OR, USA, (2019)

[2] Henry, C., "EchoStar buys Jupiter-3 "ultra high density satellite" from SSL," Space News, 9 August 2017.

[3] Henry, C., "Eutelsat ditches ViaSat-3 investment, buys half-terabit satellite from Thales Alenia Space," Space News, 18 April 2018.

[4] Optical Internetworking Forum, "Implementation Agreement for CFP2-Analogue Coherent Optics Module," Optical Internetworking Forum, Fremont, CA, 2016.

[5] Boroson, D., Robinson, B., Murphy, D., Burianek, D., Khatri, F., Kovalik, J., Sodnik, Z., Cornwell, D., “Overview and results of the Lunar Laser Communication Demonstration," SPIE LASE, San Francisco, CA, USA (2014)

[6] Shiratama, K., Fuse, T., Kubo-oka, T., Kunimori, H., Suzuki, K., Munemasa, Y., Takenaka, H., Kolev, D., CarrascoCasado, A., Trinh, P., Toyoshima, M., "Development status on High-Speed Laser Communication Terminal "HICALI" onboard ETS-IX," IEEE ICSOS, Portland, OR, USA, (2019)

[7] Shih, T., Guldner, O., Khatri, F., Burnside, J., DeVoe, C., Hubbard, W., Constantine, S., Torres, J., Robinson, B., “A Modular, Agile, Scalable Optical Terminal Architecture for Space Communications”, IEEE ICSOS, Naha, Okinawa, Japan, (2017)

[8] Cybel LLC, STINGRAY-BT-1550 Datasheet, Bethlehem PA, USA, (2017)

[9] El-Halafawy, F., Aly, M., and El-Bary, M., "Four-Wave Mixing Crosstalk in DWDM Optical Fiber Systems," Proceedings of the Twenty Third National Radio Science Conference (NRSC'2006), Menoufiya, (2006)

[10] Miller, E., "Link geometry analysis and requirements derivation for intersatellite crosslinks within LEO broadband constellations," IEEE ICSOS, Portland, OR, USA, (2019)

[11] NASA, "Population Density - NASA Earth Observations", https://neo.sci.gsfc.nasa.gov/view.php?datasetId=SEDAC_POP (15 Jan 2021) 